\title{
First result of large size Scintillating Glass GEM imager
}

\author{
Takeshi Fujiwara $^{1, a}$, Yuki Mitsuya ${ }^{2}$, Hiroyuki Takahashi ${ }^{2}$, and Hiroyuki Toyokawa ${ }^{1}$ \\ ${ }^{1}$ National Institute of Advanced Industrial Science and Technology (AIST), Research Institute for Measurement and Analytical \\ Instrumentation, 1-1-1 Umezono, Tsukuba, Ibaraki, 305-8568 Japan \\ ${ }^{2}$ The University of Tokyo, Japan, Department of Nuclear Engineering and Management, 7-3-1 Hongo, Bunkyo, Tokyo, 113-8656 \\ Japan
}

\begin{abstract}
A large size x-ray imaging gaseous detector, which has $280 \times 280 \mathrm{~mm}^{2}$ effective area has been successfully developed and $\mathrm{x}$-ray imaging has been demonstrated. The imaging system consists of a chamber filled with $\mathrm{Ne} / \mathrm{CF}_{4}$ scintillating gas mixture, inside of which Glass GEM (G-GEM) is mounted for gas multiplication. In this system electrons are generated by the reaction between $\mathrm{x}$-rays and the gas, and visible photons by excited $\mathrm{Ne} / \mathrm{CF}_{4}$ gas molecules during the gas electron multiplication process in the G-GEM holes. These photons are simply detected with CCD-camera and a radiograph is formed. Here, we report on the properties of large size scintillation G-GEM and the results of using it as a digital x-ray imager with a large sensitive area.
\end{abstract}

\section{Introduction}

Gaseous detectors have been playing important role for various types of radiation measurements. Gas electron multiplier (GEM) is one of the most popular and successful types of micro-patterned gaseous detector (MPGD). [1-4] However, building a large size detector with MPGDs needs a lot of effort to install and maintain. In addition, signal readout is another big issue for operating MPGDs. For imaging with GEMs, readout pads needs to be carefully designed and so as the many channels of readout circuits which goes up to hundreds to thousands of channels. On the other hand, not all radiation measurements are required to be precisely measured. For instance, xray radiograph can be formed with simple integrating circuits such as CCDs and CMOS. [5, 6] From that point of view, gaseous scintillation with GEMs has been studied in past works, in order to simplify the signal readout for imaging. [7-10] Scintillation light caused during electron avalanche process with scintillation gas (such as $\mathrm{CF}_{4}$ ) can be easily detected with optical camera. However, amount of photons produced is limited with conventional GEM's avalanche process and it is difficult to be detected with conventional optical camera. This is mostly due to the gas gain is not enough with single GEM $\left(\approx 10^{3}\right)$. On the other hand, we have been developing Glass GEMs (GGEM), which is promising electron multiplier with high gas gain and low charge ups [11, 12]. Gas gain of GGEM reached up to $10^{5}$ with a single G-GEM structure may contribute to high scintillation yield. In addition, we have fabricated large size G-GEM with $300 \times 300 \mathrm{~mm}^{2}$ glass substrate, which has $280 \times 280 \mathrm{~mm}^{2}$ effective area [13]. In this paper, we report on the development of large

\footnotetext{
ae-mail: fujiwara-t@aist.go.jp
}

area x-ray imager using G-GEM and gaseous scintillation. Development of our first large-area Scintillating G-GEM detector is shown. We indicate the measured results of gas gain and optical signal to show that it worked properly. We then show the first result of gain $\mathrm{x}$-ray imaging result with large-area Scintillating G-GEM.

\section{Properties of large size G-GEM and initial experiment}

\subsection{Principle of the setup}

We have fabricated large size G-GEM using $300 \times 300$ $\mathrm{mm}^{2}$ photo-etchable glass substrate, using fabrication process of HOYA Corporation. This glass is called PEG3, and the G-GEM has $280 \times 280 \mathrm{~mm}^{2}$ effective area. Properties of the large are G-GEM especially focusing on comparison with G-GEM fabricated in our past works [10, 12] which has $100 \times 100 \mathrm{~mm}^{2}$ effective area are shown in table 1 . The outlook of the large area G-GEM is shown in Figure 1. As seen in the figure, the G-GEM are stiff enough and has a self-supporting structure, which results in easy to handle and needs no support for mounting.

On the other hand, $\mathrm{CF}_{4}$ gas is known as a scintillation gas, and to achieve high enough yield of scintillation photons for imaging, gas gain needs to be high enough also. The mechanism of scintillation light yield during GGEM's avalanche process is shown in Figure 2. $\mathrm{Ne} / \mathrm{CF}_{4}$ gas mixture is attractive gas for high gas amplification with low applying voltage. In this study, we first studied gain properties and scintillation light from $\mathrm{Ne} / \mathrm{CF}_{4}$ gas for $\mathrm{x}$-ray imaging. 
Table 1. Comparison with past reported Glass GEM and large size Glass GEM fabricated in this study.

\begin{tabular}{|c||c|c|}
\hline & $\begin{array}{c}\text { Past reported } \\
\text { G-GEM[10, 12] }\end{array}$ & $\begin{array}{c}\text { Large size } \\
\text { G-GEM }\end{array}$ \\
\hline \hline Effective area & $100 \mathrm{~mm}$ square & $280 \mathrm{~mm}$ square \\
\hline Substrate size & $145 \mathrm{~mm}$ square & $300 \mathrm{~mm}$ square \\
\hline Hole size & $180 \mu \mathrm{m}$ & $170 \mu \mathrm{m}$ \\
\hline Hole pitch & $280 \mu \mathrm{m}$ & $280 \mu \mathrm{m}$ \\
\hline Substrate thickness & $680 \mu \mathrm{m}$ & $700 \mu \mathrm{m}$ \\
\hline Number of holes & 141,417 & $1,154,423$ \\
\hline
\end{tabular}

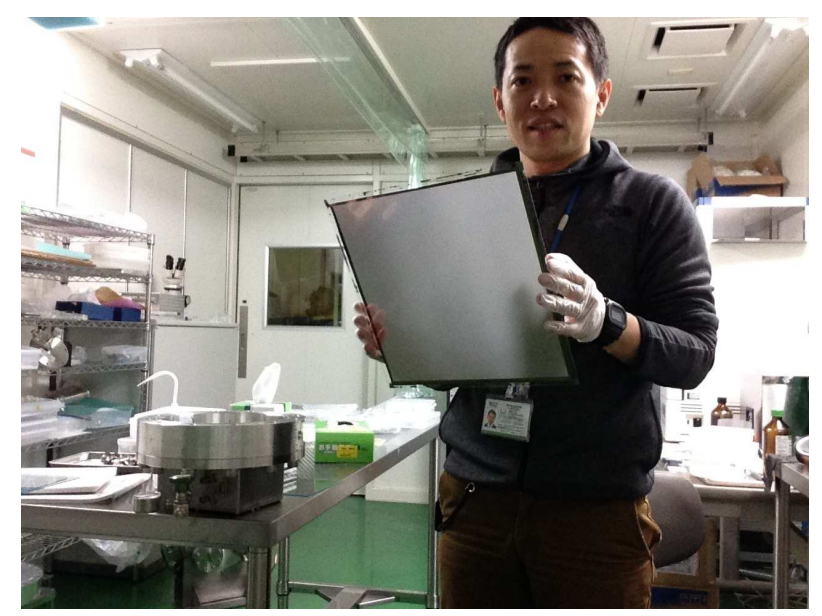

Figure 1. Outlook of the large size G-GEM $\left(280 \times 280 \mathrm{~mm}^{2}\right.$ effective area), fabricated with $300 \times 300 \mathrm{~mm}^{2}$ photo-etchable glass substrate. As the G-GEM is $700 \mu m$ thick, and has a perfectly self-supporting structure.

\subsection{Gain properties with $\mathrm{Ne} / \mathrm{CF}_{4}$ gas and Scintillation light measurement}

In order to investigate scintillation light from G-GEM with $\mathrm{Ne} / \mathrm{CF}_{4}$ gas, initial experiments were carried out using ${ }^{55} \mathrm{Fe}$ source and photo multiplier tube (PMT). Figure 3 is the experimental setup. We installed a G-GEM in a gas filled chamber with drift cathode $10 \mathrm{~mm}$, and a readout 2 $\mathrm{mm}$ gap from the optical window which plays a role as a readout anode. The chamber was flushed with an $\mathrm{Ne} / \mathrm{CF}_{4}$ (90:10) gas mixture at $50 \mathrm{~mL} / \mathrm{min}$ of flow rate. High voltages to the G-GEM's electrodes were applied with individual voltage supply. The drift field was $1 \mathrm{kV} / \mathrm{cm}$, and induction field was $2 \mathrm{kV} / \mathrm{cm}$. In this setup, charge and scintillation light produced with G-GEM were read at the same time using two MCAs. First, charge signal was read out from a charge sensitive preamplifier connected to the anode electrode placed in the bottom of G-GEM. The output from charge sensitive amplifier output was connected to a shaping amplifier and a MCA for taking pulse height spectra (figure 4). At the same time, scintillation light from G-GEM were detected with PMT placed right under the chamber. PMT was also connected to charge sensitive amplifier, shaping amplifier and MCA, and pulse height spectra of ${ }^{55} \mathrm{Fe}$ were recorded at the same time. The spectra obtained in this measurement in Figure 5. Due to the efficiency of light collecting was not good enough in

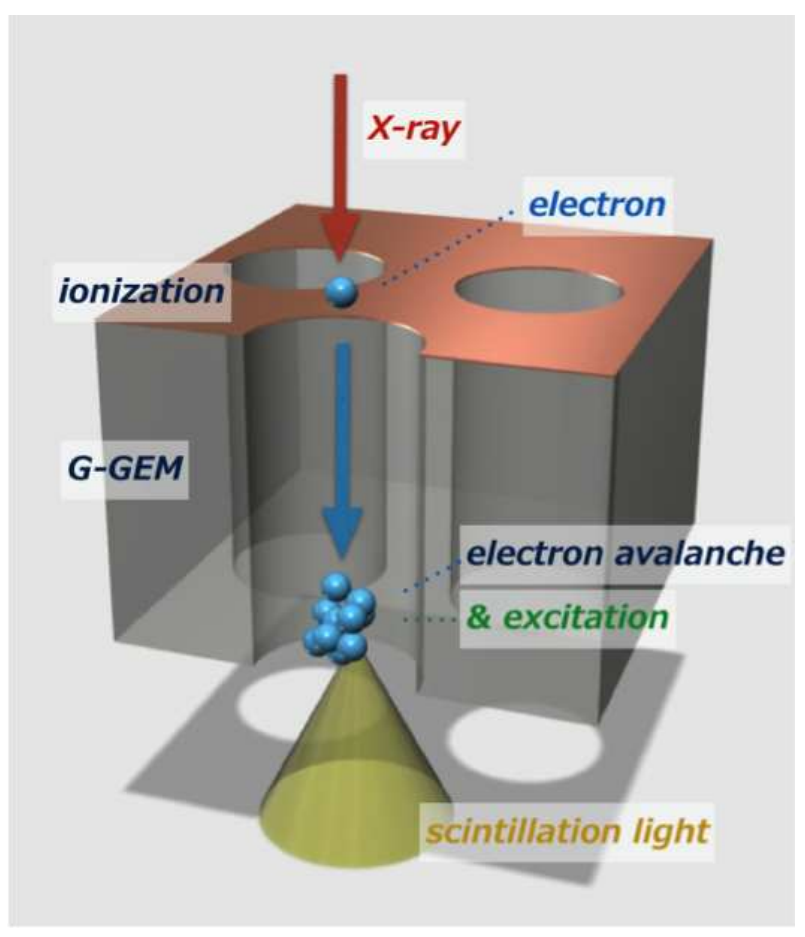

Figure 2. Diagrammatic sketch of scintillation light yield from G-GEM hole during electron avalanche process in scintillation gas.

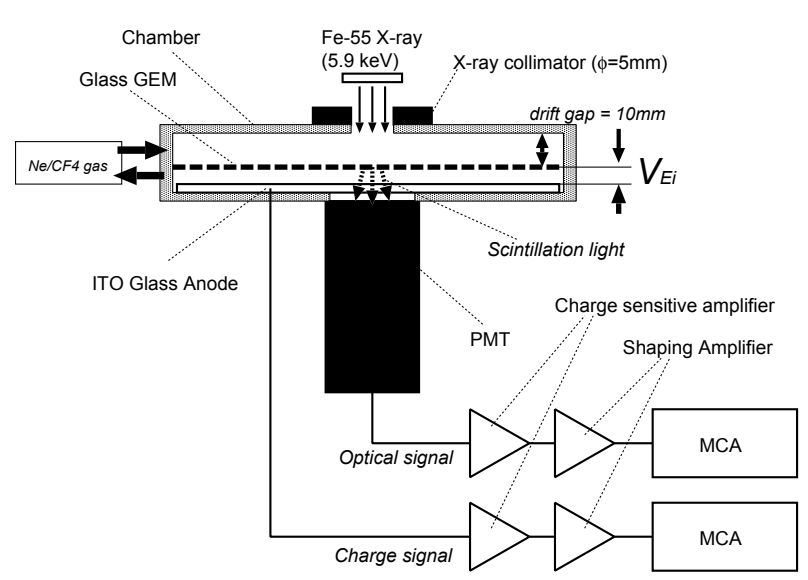

Figure 3. Experiment setup for measuring the basic performance of the scintillating G-GEM detector coupled with the photomultiplier tube (Hamamatsu R329) are described. ${ }^{55} \mathrm{Fe}$ x-ray (5.9 $\mathrm{keV}$ ) source was used in this study, and the x-ray was collimated to $\phi=5 \mathrm{~mm}$ at the entrance window of the chamber. Both the charge and the optical signal were recorded with MCAs.

this setup, we could not observe clear peak of $5.9 \mathrm{keV} \mathrm{x}$ ray, beside the energy spectra obtained with charge signal. However, the result indicates high yield of scintillation photons were produced with G-GEM and $\mathrm{Ne} / \mathrm{CF}_{4}$ gas. Gas gain curve of this G-GEM is shown in Figure 6. Gas gain reached up to $7 \times 10^{3}$ stably with single G-GEM and $\mathrm{Ne} / \mathrm{CF}_{4}$ gas. 


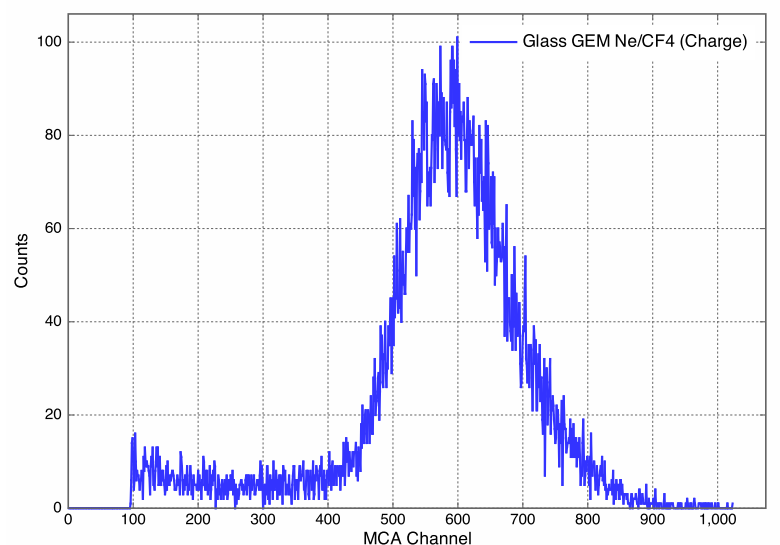

Figure 4. Measured pulse height spectrum with ${ }^{55} \mathrm{Fe} X$-ray source $(5.9 \mathrm{keV})$. Energy spectrum of charge readout is shown. Energy resolution is $29 \%$ in FWHM.

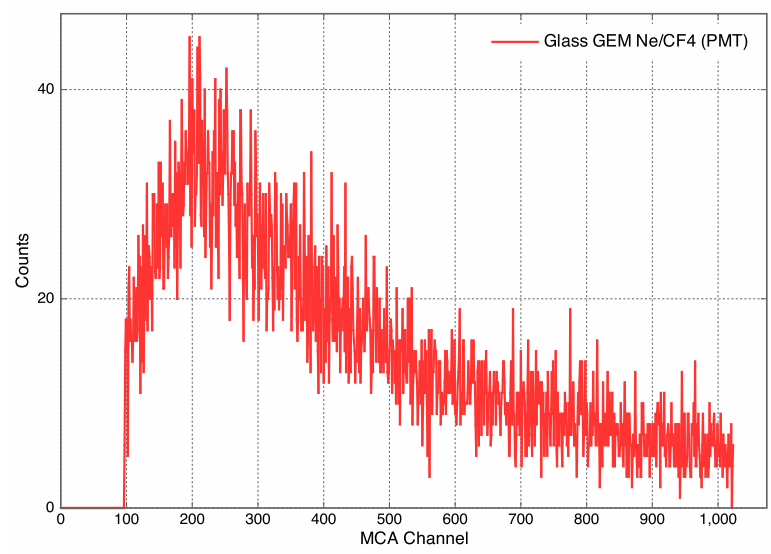

Figure 5. Energy spectrum taken at the same time using a photomultiplier tube directly coupled under optical window placed in the bottom of the gas chamber.

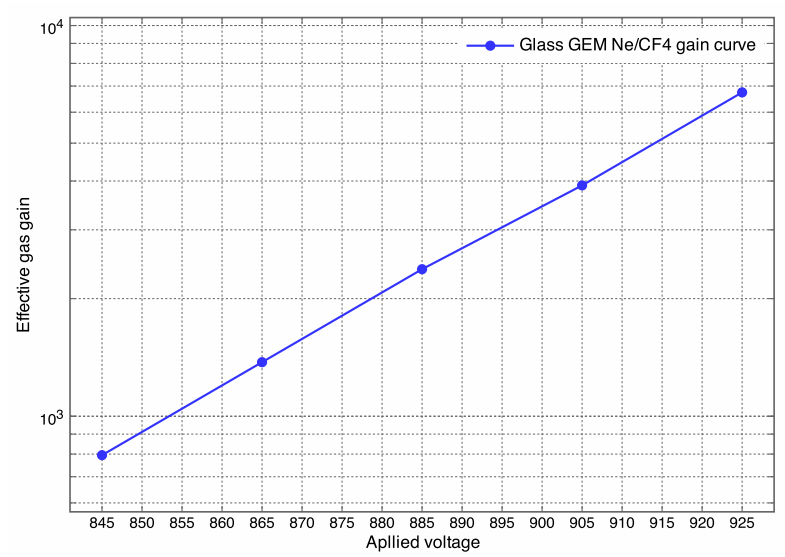

Figure 6. Gas gain curve achieved with G-GEM and Ne/CF4 (90:10) gas. Maximum gain reached up tp $7 \times 10^{3}$, applying 925 $\mathrm{V}$ to a single G-GEM.

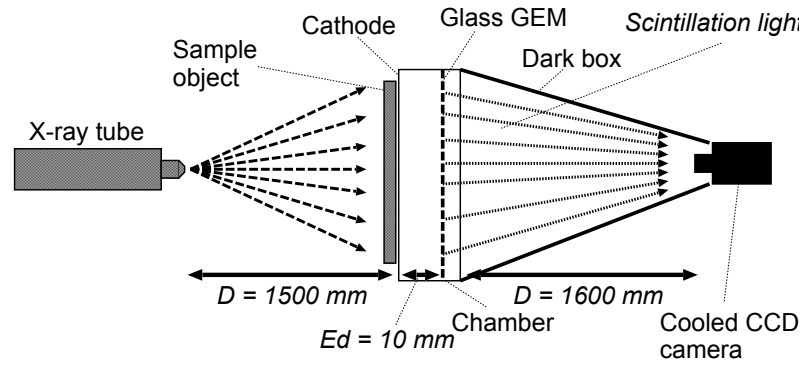

Figure 7. Schematic and detection principle of scintillating GGEM detector. Electrons created in reaction with $\mathrm{x}$-rays are multiplied by the G-GEM in the chamber. Scintillation light emitted during the electron avalanche process is detected with a CCD camera placed behind the chamber, and form an x-ray transmission image.

\section{Imaging test of large size Scintillating Glass GEM}

Figure 7 illustrates the schematic and detection principle detection principle of $x$-ray imaging with a scintillating GGEM detector. For the x-ray window of the chamber, a 20 $\mu \mathrm{m}$ thick Kapton film coated with $20 \mathrm{~nm}$ Aluminum layer was used. In this experiment, gas gain of G-GEM were set to approximately $3 \times 10^{3}$. Using this setup, we investigated the first $\mathrm{x}$-ray imaging test with the large size scintillating G-GEM detector. Detailed experimental conditions and equipment used in this study is shown in table 2. The sample object for demonstating $\mathrm{x}$-ray imaging is shown inf figure 8 . A tool box made out of plastics are filled with screws and connectors. X-ray tube were placed in front of gas chamber, and in the same line, optical cooled CCD camera (Bitran BH-50L) were placed behind the chamber. Camera is looking towards the optical window of the chamber surrounded with dark box. First demonstrating $\mathrm{X}$-ray transmission image taken by large size Scintillating G-GEM is shown in figure 9. X-ray image of $280 \times 280$ $\mathrm{mm}^{2}$ are successfully taken in 5 seconds of exposure time with simple optical camera.

Table 2. Detailed setup and equipment used in imaging test with large size scintillating G-GEM Detector

\begin{tabular}{|c|c|}
\hline Electron multiplier & $280 \mathrm{~mm}$ square G-GEM \\
\hline Scintillation gas & $\mathrm{Ne} / \mathrm{CF}_{4}(90: 10)$ \\
\hline Gas gain & $3 \times 10^{3}$ \\
\hline Optical camera & BITRAN BH50L $(300,000$ pixels $)$ \\
\hline Lens & Fujinon $25 \mathrm{~mm} \mathrm{F0.85}$ \\
\hline x-ray source & Amptek Mini-x $(25 \mathrm{kV}, 50 \mu \mathrm{A})$ \\
\hline
\end{tabular}

\section{Summary}

A large size scintillating G-GEM detector that $280 \times 280$ $\mathrm{mm}^{2}$ in size was developed and operated successfully. The gain properties and gas scintillation yield with $\mathrm{Ne} / \mathrm{CF}_{4}$ gas were quantitatively studied, and succeeded in achieving fine X-ray transmission images with the scintillating 


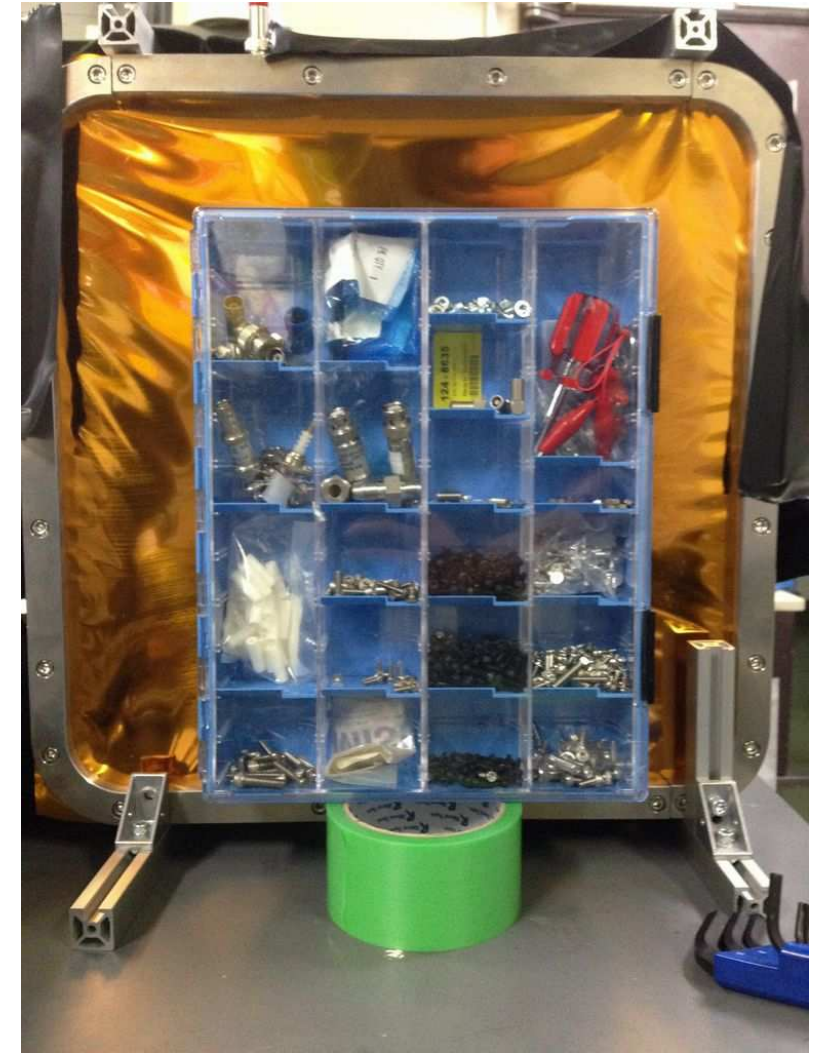

Figure 8. A photograph of tool box filled in with screws an connectors, used as a sample object for $\mathrm{x}$-ray imaging demonstration of scintillating G-GEM detector.

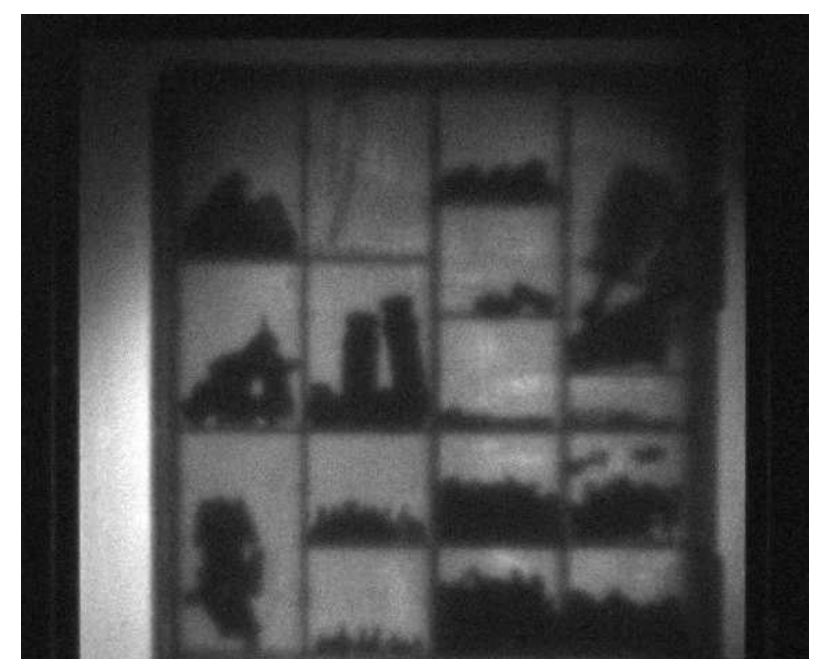

Figure 9. X-ray transmission image of a tool box obtained by large size scintillating G-GEM detector with an x-ray tube voltage of $25 \mathrm{kV}$, current $50 \mu \mathrm{A}$. Effective area of the $\mathrm{x}$-ray image is $280 \times 280 \mathrm{~mm}^{2}$. The image was taken with an exposure time of 5 seconds (gas gain: 3,000 ).
G-GEM detector. Using the high yield photon emission from the $\mathrm{Ne} / \mathrm{CF}_{4}$ gas mixture, the scintillation light from the high gain G-GEM can be easily detected with a commercially available CCD camera coupled to a conventional lens system. Although the data acquisition rate is restricted due to the speed of the CCD readout, the optical readout is very simple and attractive. Moreover, the CCD camera can provide fine imaging due to the very large number of channels (up to $3 \times 10^{6}$ ). Therefore, we conclude that our detector has the potential to be an alternative to flat-panel detectors, imaging plates, x-ray films, and CMOS sensors for digital $x$-ray imaging.

\section{Acknowledgement}

The authors wish to acknowledge Takashi Fushie (HOYA Corporation), an expert on fabricating Glass GEMs with photo-etchable glass, for his great help in interpreting the significance of the results of this work.

\section{References}

[1] Sauli F 1997 GEM: A new concept for electron amplification in gas detectors Nuclear Instruments and Methods in Physics Research Section A: Accelerators, Spectrometers, Detectors and Associated Equipment 386 $531-4$

[2] Sauli F 2016 The gas electron multiplier (GEM): Operating principles and applications Nuclear Instruments and Methods in Physics Research Section A: Accelerators, Spectrometers, Detectors and Associated Equipment 805 2-24

[3] Breskin A, Alon R, Cortesi M, Chechik R, Miyamoto J, Dangendorf V, Maia J M and Santos Dos J M F 2009 A concise review on THGEM detectors Nuclear Instruments and Methods in Physics Research Section A: Accelerators, Spectrometers, Detectors and Associated Equipment 598 107-11

[4] Coimbra A E C, Breskin A and Santos Dos J M F 2012 THGEM operation in high pressure Ne/CF 4 J. Inst. 7 C02062-2

[5] Lumb, D.H.; Nousek, J.A., "Energy and time response of CCD X-ray detectors," in Nuclear Science, IEEE Transactions on , vol.39, no.5, pp.1379-1383, Oct 1992

[6] Cho, Min Kook; Kim, Ho Kyung; Graeve, T.; Seung Man Yun; Lim, Chang Hwy; Dong Hyun Cho; Kim, J.-M., "Measurements of X-ray Imaging Performance of Granular Phosphors With Direct-Coupled CMOS Sensors," in Nuclear Science, IEEE Transactions on , vol.55, no.3, pp.1338-1343, June 2008

[7] Fraga F, Margato L, Fetals S, Fraga M, Marques R and Policarpo A 2003 Luminescence and imaging with gas electron multipliers Nuclear Instruments and Methods in Physics Research Section A: Accelerators, Spectrometers, Detectors and Associated Equipment 513 379-87

[8] Fraga F A F, Margato L M S, Fetal S T G, Fraga M M F R, Ferreira Marques R and Policarpo A J P 
L 2001 Optical readout of GEMs Nuclear Instruments and Methods in Physics Research Section A: Accelerators, Spectrometers, Detectors and Associated Equipment 471 125-30

[9] Fraga F A F, Fetal S T G, Margato L M S, Ivaniouchenkov I, Marques R F and Policarpo A J P L 1999 Imaging detectors based on the gas electron multiplier scintillation light 1999 IEEE Nuclear Science Symposium. Conference Record. 1999 IEEE Nuclear Science Symposium and Medical Imaging Conference vol 2 (IEEE) pp 829-33

[10] Fujiwara T, Tanaka S, Mitsuya Y, Takahashi H, Tagi K, Kusano J, Tanabe E, Yamamoto M, Nakamura N, Dobashi K, TOMITA H and Uesaka M 2013 Development of a scintillating G-GEM detector for a 6-MeV X- band Linac for medical applications J. Inst. 8 C12020-0

[11] Takahashi H, Mitsuya Y, Fujiwara T and Fushie T 2013 Development of a glass GEM Nuclear Instruments and Methods in Physics Research Section A: Accelerators, Spectrometers, Detectors and Associated Equipment 724 1-4

[12] Fujiwara T, Mitsuya Y, Takahashi H, Fushie T, Kishimito S, Guerard B and Uesaka M 2014 The performance of Glass GEM J. Inst. 9 P11007-7

[13] Mitsuya Y, Fujiwara T, Fushie T, Maekawa T and Takahashi H 2015 Development of large-area glass GEM Nuclear Instruments and Methods in Physics Research Section A: Accelerators, Spectrometers, Detectors and Associated Equipment 795 156-9 